\title{
SALUD INTERNACIONAL: EL NUEVO DESAFÍO PARA LA EDUCACIÓN DE ENFERMERÍA
}

\author{
Maria da Gloria Miotto Wright* \\ Denise M. Korniewicz** \\ Charles Godue*** \\ Maricel Manfredi ${ }^{* * * *}$
}

WRIGHT, M.da G.M.; KORNIEWICZ, D.M; GODUE, C.; MANFREDI, M. Salud internacional: el nuevo desafío para la educación de enfermería. Rev.latino-am.enfermagem, Ribeirão Preto, v. 6, n. 3, p. 5-10, julho 1998.

Para identificar las actividades de salud internacional (SI) en escuelas de enfermería y analizarlas en forma preliminar, se utilizó una muestra de 110 escuelas norteamericanas y, paralelamente, se enviaron cuestionarios a 4 escuelas latinoamericanas y a una del Caribe (ALC). Parte significativa de las escuelas norteamericanas y todas las 5 escuelas de ALC tienen actividades de salud internacional. Dicho interés es básicamente un fenómeno de los últimos cinco a dez años. Hubo diferencias en la estructura de las actividades de SI entre las escuelas, pero el tipo de apoyo ofrecido para actividades internacionales era similar, tanto en los EEUU como en ALC. El componente de SI en el currículo de las escuelas norteamericanas estaba dentro de cursos sobre cultura, sistemas de salud y salud de la comunidad, en cuanto en las escuelas de ALC dicho componente estaba en cursos relacionados con promoción de la salud, políticas y estrategias de salud y perspectivas de enfermería. Esas escuelas tienen sólamente $10 \%$ de sus profesores y estudiantes involucrados en actividades de salud internacional. Se verificó que las escuelas de enfermería todavía no hacen parte de los cursos y actividades que las Escuelas de Salud Pública han implementado en ese campo y se observan áreas que deben ser fortalecidas para que la enfermería ocupe una posición de liderazgo en la práctica y la investigación en salud internacional.

TÉRMINOS CLAVES: salud internacional, educación de enfermería, América Latina y Caribe, Estados Unidos, proyectos internacionales

\section{INTRODUCCIÓN}

Las dimensiones ideológicas, políticas y económicas de la salud son asuntos internacionales. Los sectores social, económico y de la salud son influenciados por la interdependencia y globalización de la economía mundial. El desarrollo económico y de la salud están crecientemente interligados, obligando a varios gobernantes a revisar el papel del Estado como un proveedor de servicios a la población. Como resultado, se está creando una consciencia internacional de la necesidad de mejorar la salud y el bienestar de la población como un de los requisitos para la paz y solidariedad entre las naciones (FRENK \& CHACÓN, 1992; CONTANDRIOLOULOS, 1992; BUSTELLO, 1992; PAHO, 1992).

El aumento de la complejidad e importancia de las relaciones internacionales estas elevando el campo de salud internacional a una posición de destaque como un área de estudio, práctica y de investigación. Las características de los problemas de salud no reconocen las fronteras nacionales; al contrario, pueden abrir o reforzar áreas de cooperación o, en otra ocasiones, crear conflictos en la comunidad internacional. La interrelación del campo de salud internacional con el desarrollo de un país enfatiza la salud internacional como un instrumento o una meta para disminuir el espacio entre la universidad, la sociedad y la población. También introduce, el enfoque interdisciplinario como una estrategia para alcanzar los resultados esperados en las áreas de educación y salud.

El nuevo modelo reconoce la diversidad económica, política y social entre los países, así como la manera que estos aspectos condicionan el proceso de desarrollo y salud de la población.

De acuerdo con PANISSET (1992) y ROVERE (1992), salud internacional está en dos dimensiones de la

\footnotetext{
* R.N., M.S., Ph.D. Escuela de Enfermería, Universidad de Georgetown, Washington, D.C./USA. Universidad de Brasilia/Brasil (de licencia)

** R.N., DscN, FAAN. Escuela de Enfermería, Universidad de Georgetown, Washington, D.C.

*** M.D. Organización Panamericana de la Salud, Washington, D.C.

**** R.N., MscN. Organización Panamericana de la Salud, Washington, D.C.
} 
salud: (i) salud como asunto de las relaciones internacionales (SARI), concepto que se refiere a los determinantes políticos, económicos y militares al nivel internacional y que directa o indirectamente afectan la salud de la población; y (ii) la dimensión internacional de la salud (DIS), que se refiere a los condicionantes de la salud, como la internacionalización de los riesgos, de los modelos de los servicios de salud, de la transferencia de ciencia y tecnología de un país a otro, y del modelo de formación de recursos humanos. Dichos fenómenos están en el espacio nacional de cada país y afectan directa o indirectamente la salud de la población.

Los conceptos de desarrollo y proceso dinámico fueron incorporados en las dos dimensiones de la salud presentados por PANISSET (1992); ROVERE (1992) y WRIGHT (1995), cuando introduce el concepto de salud internacional como "un proceso dinámico de interacción, integración, y equilibro de poder, interés y toma-dedecisión de la comunidad internacional y nacional en relación a asuntos y problemas de salud a nivel nacional e internacional con repercusión en sus relaciones con el desarrollo del país y del mundo". Autores como FRENK \& CHACÓN (1992) consideran la salud internacional como un campo de estudio, desarrollo de conocimientos y de práctica. NEUFELD (1992), afirma que salud internacional involucra aspectos "relacionados con salud y desarrollo; basados en valores de equidad y beneficios mutuos, donde el análisis es específico para una entidad geopolítica y los resultados sirven para hacer comparaciones entre países y apoyar la toma de decisiones a nivel nacional e internacional". GODUE (1996) considera que "salud internacional es un campo de estudio que involucra esfuerzos deliberados para mejorar la salud de la población según el interés nacional basados en las funciones analíticas y operacional de los gobiernos de cada país". PANISSET (1996), enfoca la "salud internacional cómo un área de estudios dentro del campo de relaciones internacionales, con el fin de entender como los sistemas de intercambio comercial, intelectual, social y política afectan la condición de salud de las poblaciones".

\section{OBJETIVO}

La universidad deberá integrarse a ese panorama, ofreciendo programas novedosos para los futuros profesionales. Se necesita educar y entrenar los enfermeros para enfrentar estos nuevos desafíos. Actualmente existen pocas investigaciones sobre cómo el currículo de enfermería y las actividades asociadas a los cursos preparan a los enfermeros para trabajar en salud internacional y tratar de asuntos vinculados a la salud internacional. Como parte del proceso de desarrollo de la enfermería en dicho campo, la Escuela de Enfermería de la Universidad de Georgetown en Washington, D.C., conjuntamente con la Organización Panamericana de Salud y la Organización Mundial de la Salud (OPS/OMS), condujeron el primer estudio de una muestra de escuelas de enfermería en los Estados Unidos, América Latina y el Caribe. El objetivo fue identificar la situación curricular y actividades relacionadas con salud internacional en las escuelas de enfermería seleccionadas.

\section{PROCEDIMIENTOS}

Se quería identificar la naturaleza de las actividades que hay en las escuelas de enfermaría en salud internacional, sujeto a las limitaciones de recursos para la encuesta. Así, se buscó maximizar la información que se podría obtener bajo la restricción presupuestal. Así, se seleccionaron 10 escuelas norteamericanas en que OPS/OMS había identificado dichas actividades: 7 Centros Colaboradores de Enfermería de la OMS en los EEUU (de las cuales 5 respondieron) y tres escuelas de enfermería de la región metropolitana de Washington, D.C.. Como no se sabía a priori cuáles de las otras escuelas norteamericanas de enfermería tenían actividades internacionales, se trató de aumentar el número de experiencias mediante la selección al azar 100 de escuelas norteamericanas adicionales de un listado de las 339 escuelas de enfermaría cuyos programas son ofertados por universidades. El resultado sobreestimará ligeramente el porcentaje de escuelas norteamericanas que tienen actividades internacionales, lo que es coherente con el objetivo de obtener el número máximo de experiencias y no la representatividad estadística.

Como no era viable realizar en semejante escala una investigación en Latinoamérica y el Caribe (ALC), se seleccionaron apenas 5 escuelas (una en cada pais de Brasil, Colombia, Jamaica, México y Nicaragua) seleccionadas por OPS por tener ex-residentes de su programa de entrenamiento en salud internacional y desarrollar actividades en esa área. Se buscó con esa muestra, (a pesar de pequeña y no representativa), información que podría ser útil en entender cómo escuelas que tenían interés en salud internacional estructuraban sus actividades y si había experiencias cualitativamente diferentes o innovadoras en esas escuelas.

Así, se envió un cuestionario con 16 preguntas por fax o correo a 115 escuelas de enfermería, siendo 110 norteamericanas y 5 de ALC. Se incluyó una carta que explicó los objetivos del estudio. Hubo seguimiento por teléfono de cuestionarios que no fueron devueltos puntualmente para aumentar el número de respuestas.

El cuestionario abarcó los siguientes aspectos: el 
tipo de programas y proyectos de SI que la Escuela tiene; el interés de la escuela de enfermería en salud internacional (SI); la estructura de la SI en la escuela; el tipo de apoyo que la Escuela ofrece para las actividades de SI; y la participación de estudiantes y profesores en SI. Los datos fueron recolectados en Febrero y Marzo de 1995 y los resultados preliminares fueron presentados en el I Taller Latinoamericano y Caribeño de Salud Internacional, realizado en la Universidad de Georgetown, Washington, D.C., entre 7-9 de junio de 1996, con el soporte técnico y financiero de la OPS/OMS.

\section{RESULTADOS}

De las 115 escuelas a que se enviaron cuestionarios, 72 respondieron, compuestas por todas las 5 escuelas de ALC, 59 de las 100 escuelas estadounidenses seleccionadas al azar, todas las 3 escuelas de la región de Washington-Baltimore, y 5 de los 7 Colaboradores de la OMS en EEUU.

Solamente 43 de las 59 escuelas norteamericanas que respondieron poseen algún tipo de actividad internacional. Todas las 5 escuelas ALC tienen actividades SI, como había sido indicado por OPS.

Los resultados serán presentados de acuerdo con las categorías: (i) salud internacional y la educación de enfermería; (ii) salud internacional y la práctica de enfermería; (iii) salud internacional y la investigación en Enfermería.

\section{(i) Salud internacional y la educación de enfermería}

Los siguientes aspectos fueron identificados:

- 43/59 de las escuelas norteamericanas y las 5 de ALC tiene interés en salud internacional y tienen algún tipo de actividad en el área.

- Sólamente 22\% (13/59) de las escuelas norteamericanas y $40 \%(2 / 5)$ de ALC tienen salud internacional entre las prioridades generales de la escuela de enfermería.

- En algunas escuelas de enfermería las actividades de SI tenían cierta estructura y en otras escuelas se desarrollaban informalmente. En las escuelas norteamericanas, la estructura más común era el Centro (18\%, o 8/43, incluyendo los 5 Centros Colaboradores de Enfermería de la OMS que respondieron el cuestionario) y el programa o grupo de trabajo $(16 \%$, o $7 / 43)$. Para las escuelas de ALC, la estructura más común era también un programa o grupo de trabajo $(60 \%$, o $3 / 5)$.

- El tipo de apoyo que las escuelas dan, para el área de salud internacional es similar en EEUU y ALC. En ambas regiones, el interés en salud internacional es un fenómeno reciente (dentro de los últimos cinco a diez años).
- Las estrategias utilizadas en EEUU y ALC para integrar salud internacional al currículo de enfermería varían desde un único curso hasta un área o unidad concentrada dentro de un programa; hay también casos de un curso ofrecido conjuntamente por dos departamentos. En los EEUU, salud internacional está relacionada más con aspectos culturales, sistemas de salud y salud de la comunidad; en ALC, con la promoción de la salud, políticas de salud y perspectivas de enfermería.

- La manera que se involucran los estudiantes y profesores en actividades de salud internacional es similar en EEUU y ALC. Las actividades normalmente son de carácter individual y no representan un esfuerzo sustancial por parte de las escuelas.

- El 70\% (30/43) de las escuelas norteamericanas y todas las 5 de ALC que tiene SI tiene menos de $10 \%$ de sus profesores y estudiantes involucrados en actividades de SI.

\section{(ii) Salud internacional y la práctica de enfermería}

El estudio identificó las formas que utilizaban las escuelas de enfermería para integrar la perspectiva de salud internacional en la práctica de enfermería, las cuales consisten de programas de estudiantes y proyectos de profesores que se desarrollan fuera del país de origen.

\section{- Programas internacionales para los estudiantes}

La mayoría de los programas internacionales de los estudiantes de EEUU y ALC son desarrollados informalmente y normalmente no implican en acuerdo formal entre los países e instituciones involucradas.

\section{- Aspectos generales de los programas de estudiantes de EEUU}

- 31 escuelas de enfermería ofrecen programas internacionales para sus estudiantes (involucran actividades fuera del país).

- La mayoría de dichos programas se iniciaron en la década de 1990 y tienen una duración entre 10 días a un semestre.

- Los programas internacionales de los estudiantes son integrados dentro del currículo de enfermería a través de cursos electivos, cursos esporádicos, experiencia de viaje del último año del curso, experiencia clínica en otro país, o como estudio independiente.

- Las regiones del mundo utilizadas para desarrollar estos programas internacionales son: Europa Occidental (más común), América Latina, Africa, Oriente Medio, Europa Oriental. 
- Aspectos generales de los programas de estudiantes de ALC

- Las escuelas de ALC ofrecen programas nacionales con trabajos en comunidades, con el apoyo de la universidad o del gobierno. Estos programas incluyen trabajos voluntarios de los estudiantes con diferente clases sociales y culturales $\mathrm{y}$, a veces, experiencias en otras regiones del mismo país.

- Dos de las 5 escuelas ALC permiten que sus estudiantes hagan el postgrado (Maestría o Doctorado) en el exterior (no necesariamente en salud internacional). Solamente una permite que estudiantes de pregrado participen de experiencia práctica fuera del país.

- Un motivo porque las escuelas ALC no promueven más programas de estudiantes en el exterior es la gran diversidad de experiencias hay dentro de cada uno de los países.

\section{- Proyectos internacionales de profesores}

Los proyectos de los profesores de EEUU y ALC son generalmente desarrollados informalmente, sin acuerdo formal entre países e instituciones.

\section{- Proyectos norteamericanos}

- 34 escuelas de enfermería de EEUU tenían proyectos internacionales con participación de profesores.

- 24 de dichas escuelas iniciaron sus proyectos en la década de 1990 y 4 los iniciaron en la década de 1980; las otras no suministraron información sobre el tema.

- La mayoría de los proyectos tenían una duración entre dos meses y un semestre.

- Los proyectos típicamente involucraron entre 1 y 4 profesores para su implementación.

Excluyendo la investigación (ver ítem iii), los 31 proyectos de los profesores de EEUU abarcaron las siguientes áreas: educación (17); servicio (9); consultoría (3); y trabajo voluntario (2).

\section{- Proyectos de América Latina y del Caribe}

- Las cinco escuelas de enfermería del ALC tenían diez proyectos internacionales con la participación de los profesores.

- Los diez proyectos tuvieron su inicio en la década de 1990.

- La mayoría de los proyectos tenían una duración entre dos meses y un año.

- Los proyectos generalmente involucraban entre cinco y diez profesores para su implementación.
Excluyendo la investigación (ver iten iii), los cinco proyectos de los profesores de ALC estaban en la área de educación.

\section{(iii) Salud internacional y la investigación en enfermería}

Hubo pocos proyectos de investigación que involucraron un esfuerzo colaborativo de los profesores de los países participantes.

- Las escuelas norteamericanas de enfermería tenían 13 proyectos de investigación desarrollados fuera de los Estados Unidos y las escuelas de ALC 5 proyectos extraregionales.

- Los profesores norteamericanos tuvieron proyectos en las siguientes áreas: (i) promoción de la salud de los de mayor edad; (ii) clasificación internacional de la práctica de enfermería; (iii) cuidado rápido del diabético; (iv) programas de salud materno-infantil; (v) estudio sobre lactancia materna; (v) la historia de la mujer en relación a la menstruación y al nacimiento de un hijo.

- Los profesores de ALC estaban interesados en desarrollar estudios de investigación relacionados con: (i) determinantes de «stress» percibido pelas madres con niños pequeños con enfermedades crónicas; (ii) metodología de integración de la enseñanza y asistencia de enfermería; (iii) modelo teórico de la asistencia de enfermería para salud pública; (iv) estudio del control de la malaria; (v) programa de intercambio académico: Cuba, Italia, México, Portugal, España.

\section{DISCUSIÓN}

Los resultados del estudio confirman las indicaciones previas de que la relación de salud internacional y enfermería es un fenómeno reciente (FENTON \& ANDERSON, 1995; CRAMAN, 1992; UHL, 1993). Parte del interés puede estar directa o indirectamente relacionado con el aumento de la interdependencia de los países y la globalización de la economía del mercado. Dichos fenómenos crean nuevas relaciones y desafíos para los políticos, administradores, educadores, profesionales y científicos, quienes buscan soluciones para los problemas de salud y desarrollo de sus países.

El estudio mostró que las actividades de salud internacional de las escuelas norteamericanas siguen las orientaciones de las escuelas de salud pública de EEUU en salud internacional, donde los proyectos de sus alumnos y profesores se direccionan a los países en desarrollo (GODUE, 1992). Esta situación es menos evidente entre los programas y proyectos de las escuelas de ALC.

A pesar del interés de las escuelas de enfermería 
de EEUU y ALC en salud internacional, el área no ha llegado a ser una prioridad del plan estratégico de desarrollo de las escuelas. Falta un apoyo expresivo de las escuelas de enfermería para las actividades de salud internacional. Esta situación es opuesta a lo que se encuentra los centros universitarios de salud internacional y las escuelas de salud pública, donde salud internacional hace parte del plan estratégico de la escuela como un todo, con presupuesto y apoyo administrativo específico (FOURNIER, 1995; GODUE, 1992; NEUFELD, 1992). Dicho fenómeno es preocupante, puesto que proyectos y programas individualizados, característicos de los de las escuelas de enfermería, no son suficientes para que los profesionales de enfermería puedan conquistar el reconocimiento de su actuación en la comunidad internacional. En este aspecto, los Centros Colaborativos de Enfermería de la OMS (WHO, 1994), son también iniciativas recientes en salud internacional (FENTON \& ANDERSON, 1995) y el resultado del estudio reveló que sus actividades en SI son similares a las de las otras escuelas de enfermería de la muestra que tienen actividades de salud internacional.

Los profesionales de enfermería todavía no están atraídos por temas o problemas de salud internacional que exija investigación para generar nuevos conocimientos. Los proyectos de enfermería enfatizan más asuntos específicos de enfermería que asuntos más amplios de salud internacional. Aunque hay un número expresivo de actividades de nivel internacional entre las escuelas de enfermería, no hay transferencia de recursos financieros entre las escuelas o entre las de EEUU y ALC para actividades colaborativas o acuerdos formales entre los países. Salud internacional es un proceso de dos caminos, de modo que la cooperación técnica y financiera debería beneficiar a la dos partes (FRENK \& CHACÓN, 1992).

Los profesores o estudiantes involucrados en programas internacionales participan básicamente de actividades educacionales, práctica, o de investigación bajo el modelo de transferencia unidireccional ("one-way transfer"), o sea, bajo la visión tradicional de salud internacional. FENTON (1994), comenta que «las enfermeras, educadores, y consultores de los Estados Unidos hemos exportado nuestras experiencias de currículo de enfermería y sus modelos a otros países, enfatizando el cuidado intensivo a nivel de hospital, porque es esto lo que sabemos hacer mejor.» Eso es realzado por lo contenido en los currículos de enfermería sobre SI: las escuelas norteamericanas introducen la perspectiva de salud internacional a través de los cursos relacionados con sistema de salud, cultura y salud de la comunidad, en cuanto en las escuelas de ALC relacionan los cursos con promoción de la salud, políticas y estrategias de salud, y perspectivas de enfermería.

\section{CONCLUSIÓN Y RECOMENDACIÓN}

El área de enfermería ha contribuido apenas marginalmente al desarrollo del campo de salud internacional. La perspectiva y las actividades de SI en las escuelas de enfermería son introducidas en la educación y práctica de enfermería solamente para enriquecer la educación general de enfermería en vez de ofrecer una educación y entrenamiento especifico en el campo de salud internacional. La enfermería todavía se encuentra, por lo tanto, en desventaja con respecto a las escuelas de salud pública en relación a recursos, planes estratégicos, conocimientos y habilidades necesarias para ejercer liderazgo en el campo de salud internacional.

Para estudiar, trabajar e investigar en SI, es necesario haber acuerdos formales entre escuelas de enfermería y universidades para implementar parcerías o asociaciones para desarrollar programas y proyectos de cooperación. Dichos programas y proyectos deberán involucrar profesores y estudiantes y beneficiar todas las partes (two-way exchange).

La introducción de la nueva perspectiva de salud internacional en el ambiente universitario ofrece una oportunidad para las escuelas de enfermería promover la educación y entrenamiento necesario para que los profesionales de enfermería puedan trabajar en el campo de salud internacional, considerada como un área de estudio, práctica e investigación. Esto permitirá la profesión de enfermería a participar en la solución de las necesidades de la sociedad del siglo entrante, además de asumir papel de liderazgo en la comunidad internacional.

Se recomiendan las siguientes medidas:

1) En los currículos de enfermería, sustituir el "Modelo Médico y el Enfoque Salud-Enfermedad" por el "Modelo de Equidad y Desarrollo y el Enfoque Interdisciplinario" (que considera el contexto de salud de las poblaciones de una perspectiva multidimensional de los aspectos socio, económico, político, cultural y espiritual.

2) Formar parcerías y asociaciones oficiales para acuerdos de cooperación técnica y financiera entre las instituciones y países involucrados en salud internacional. 3) Desarrollar nuevos conocimientos científicos de salud internacional, basados en mutua división y intercambio de ideas, recursos y tecnología.

4) Incluir en el currículo de enfermería temas relacionados a economía de la salud, políticas de salud, epidemiología social, relaciones internacionales y cooperación técnica. Con estos cambios en la formación, práctica profesional y el contexto de las investigaciones, la enfermería como un área científica tendrá los conocimientos y habilidades para enfrentar los desafíos presentados por la industria de salud y por la población. 
AGRADECIMIENTOS

Quisiéramos agradecer a las Decanas de las Escuelas de Enfermería de los Estados Unidos, América Latina y el Caribe, que muy gentilmente respondieron al cuestionario preparado para esta investigación. Agradecemos, también, a la Decana Dr. Elaine Larson, de la Universidad de Georgetown, por su apoyo durante la investigación; a la profesora Melissa Zerbe y a las alumnas Allison Morse y Wendy Cirus por sus valiosas contribuciones en todas las etapas de la investigación. Por fin, agradecemos al Programa de Desarrollo de Recursos Humanos de la OPS/OMS por su apoyo financeiro y técnico al proyecto.

\section{INTERNATIONAL HEALTH: A NEW CHALLENGE TO NURSING EDUCATION}

A survey was conducted among 110 Schools of Nursing in USA and 5 Schools in Latin America and the Caribbean (LAC) to identify the international health (IH) component in nursing education, practice and research. A significant part of U.S. schools and all 5 LAC schools have international activities, and this interest has started basically in the last 5 to 10 years. There was difference in the structure of IH activities among U.S. and LAC nursing schools, but they were similar in the type of support offered to IH initiatives. IH content in nursing education among U.S. schools was related to culture, health systems and community health; in LAC schools, IH content was related to health promotion, health policy and strategies and nursing perspectives. U.S. and LAC schools with international activities have only $10 \%$ of their faculty and students involved with IH initiatives. The nursing schools still lack courses and activities that the Schools of Public Health have implemented to deal with IH. The article observes areas that need to be strengthened so that nursing professionals can expand their leadership roles in research and practice in international health.

KEY WORDS: international health, nursing education, Latin America and the Caribbean, United States, international projects

\section{REFERÊNCIAS BIBLIOGRÁFICAS}

01. BUSTELLO, E.S. The rise of the I11-fare State: adjustment and social policy in Latin America. PAHO, n. 95, p.7-27, 1992.

02. CONTANDRIOPOULOS, A.P. Cost and equity in health system. PAHO, n. 95, p. 87-101,1992.

03. CRAMAN, A.M. Nursing within the international community: a visionary experience. Perspect.Psychiatr.Care, v. 28, n.1, p. 20-23, 1992.

04. FENTON, M.V. International affairs: Alma-Ata, at last. J.Prof. Nurs., v.10, n.1, p. 4, 1994.

05. FENTON, M.V.; ANDERSON, E.T. Nursing's international agenda: what are the WHO collaborating centers and whom do they serve? J. Prof. Nurs., v. 11, n. 1, p.3, 1995.

06. FOUNIER, P. The role of universities and the ethics of their interventions. J. Prevent. Med., v. 40, n.1, p.11-17, 1995.

07. FRENK, J; CAHCÓN, F. Conceptual bases for education and research. PAHO, n.95, p. 205-221, 1992.

08. GODUE, C. International health and schools of public health in the United States. PAHO, n.95, p.113126, 1992.

09. . International health and maternal-child health. (Mimeographo, Intern.Document of PAHO/HRDP, 1996).

10. NEUFELD, V. Training in international health: a canadian perspective. PAHO, n.95, p.193-203, 1992.
11. PANAMERICAN HEALTH ORGANIZATION. PAHO International Health: North-South debate. Washington, D.C: PAHO, 1992. (Human Resources Development Series, n. 95).

12. PANISSET, U.B. Refections on health as an international issues. PAHO, n.95, p.165-191, 1992.

13. International health statecraft: the case of Peru's 1991 cholera epidemic. Doctoral Dissertation of Philosophy. /Presented at the John Hopkins University The Paul H. Nitze School of Advanced International Studies, May, 1996/.

14. ROVERE, M. International dimension of health. PAHO, n.95, p.149-164, 1992.

15. UHL, J.E. International affairs: global perspectives of health care reform. J. Prof. Nurs., v. 9, n. 5, p. 252, 1993.

16. WORLD HEALTH ORGANIZATION/WHO COLLABORATIVE NURSIN CENTERS. Global network of WHO collaborating centers for Nursing/Midwifery development strategic plan. Preliminary Draft, n. 2. Geneva: WHO, 1994.

17. WRIGHT, M.G.M. International Health Program at Georgetown University: a Cooperative Interdisciplinary Project at Undergraduate Level - Educational Proposal. /Paper presented at I Latin American and Caribbean International Health Workshop, at Georgetown University, Washington, D.C., June 7-9, 1995/. 